\title{
An Energy-Efficient Zoning Clustering Hierarchy for WSNs
}

\author{
Hong Tong, Hui Chen, Zhaoyu Li, Shujuan Ma
}

\begin{abstract}
Focused on the limited energy characteristics of routing protocol in wireless sensor networks, we propose an Energy-Efficient Zoning Clustering Hierarchy $(\mathrm{EEZCH})$ algorithm based on LEACH and LEACH-C. In EEZCH, the monitoring area is divided into several zonings (clusters). The cluster head is selected by taking the residual energy of the nodes and their relative positions in the cluster into consideration. Compared with LEACH and LEACH-C, simulation results show that the present algorithm can effectively balance the energy consumption of the nodes, significantly extend the network lifetime.
\end{abstract}

Keywords: WSNs; energy efficiency; EEZCH; network lifetime.

\subsection{Introduction}

Wireless Sensor Networks (WSNs) is widely used in all aspects of life. WSNs are consisted of large number of small and inexpensive sensor nodes, whose energy and capability of computing and communication are usually subjected to their own hardware and software constraints. While the network topology is dynamically changed due to a dead node getting out of the network as well as a new joining in the network. Traditional routing protocols have not been suitable for sensor networks, so the routing protocols suitable for WSNs must be studied in-depth in order to save the node's energy and extend the lifetime of WSNs[1-2].

Hong Tong, Hui Chen( $\bowtie)$, Zhaoyu Li, Shujuan Ma

Chongqing Key Lab of Mobile Communications

Chongqing University of Posts and Telecommunications

Chongqing, China

e-mail:374927511@qq.com 
Clustering hierarchical routing algorithm is very effective in saving energy, improving network scalability and prolonging the network lifetime. In clustering algorithm, the nodes in the network are divided into cluster heads $(\mathrm{CH})$ and cluster members $(\mathrm{CM})$. The $\mathrm{CH}$ are responsible for aggregating the data collected by $\mathrm{CM}$, and forwarding these data to sink node directly (single hop) or via other nodes(multi hop).This approach has the advantages of reducing energy dissipation from a global perspective and enhancing system lifetime effectively [3-4], as well as enhancing resource allocation and bandwidth reusability.

In this paper, we have developed an Energy-Efficient Zoning Clustering Hierarchy (EEZCH) algorithm based on LEACH (Low-Energy Adaptive Clustering Hierarchy) and LEACH-C algorithms. The algorithm can save node's energy consumption, enhance energy efficiency and prolong network lifetime. The rest of the paper is organized as follows. Section $\Pi$ describes the existing classic protocol. Section III describes the proposed algorithm. Performance evaluation and simulation results are presented in section IV. The entire work is concluded in section V.

\subsection{Related Work}

In this section, we introduce related work in clustering algorithms for WSNs. LEACH [5] is the most classic protocol put forward by Heinzelman etc. The algorithm brings the concept of round in, and separates the operation into two phases, the setup phase and the steady state phase. In the setup phase, the nodes clustering by self-organizing and each cluster randomly select the $\mathrm{CH}$. The decision of each node whether or not to be the $\mathrm{CH}$ for the current round is made by the node randomly generating number between 0 and 1 . If the number is less than a threshold $\mathrm{T}(\mathrm{n})$, the node becomes $\mathrm{CH}$ for the current round and broadcasts message to other nodes for becoming a $\mathrm{CH}$. Other nodes choose one cluster to join in based on signal strength. In the steady state phase, $\mathrm{CMs}$ send data to their $\mathrm{CH}$ in their schedule, then $\mathrm{CH}$ transmit the aggregating data to Base Station(BS).The threshold T(n) is given by:

$$
T(n)=\left\{\begin{array}{l}
\frac{p}{1-p \times[r \bmod (1 / p)]}, n \in G \\
\text { O, other }
\end{array}\right.
$$

Where $p$ is the desired percentage of cluster heads, $r$ is the current round number; $G$ is the set of nodes that have not become a $\mathrm{CH}$ in the $1 / \mathrm{p}$ rounds. In fact $\mathrm{T}(\mathrm{n})$ is average probability that the node has not yet done the cluster head in the $r$ round. Although LEACH has many advantages, there are still many deficiencies: Randomly selecting cluster head without considering residue energy of the node may lead the node with 
low energy to be elected as the $\mathrm{CH}$, affecting the network energy efficiency. The selected cluster head distribute unevenly in the area due to their uncertain locations. The number of cluster heads is always changed in every round.

So a lot of improved algorithms have been proposed to avoid these deficiencies in literature [6-10]. LEACH-C [6] utilizes the base station (BS) for clustering. The BS receives information regarding the location and energy level of each node in the network to find a predetermined number of $\mathrm{CHs}$ and configures the network into clusters. The cluster groupings are chosen to minimize the energy required for CMs to transmit their data to their respective $\mathrm{CH}$. Though it performs better performance than LEACH, its complication and high cost could not be ignored.

\subsection{Proposed Scheme}

In this section, we introduce EEZCH which is based on LEACH and LEACH-C to save energy and maximize the network lifetime. The analysis results of LEACH and LEACH-C show that taking node's residual energy and its position into consideration can balance the energy consumption of each node, and extend network lifetime. The predetermined number of cluster heads also makes the network more stable. Therefore, this paper use the advantages of the above to propose an improved algorithm called Energy-Efficient Zoning Clustering Hierarchy (EEZCH).

\subsubsection{System Model}

$\mathrm{N}$ sensor nodes randomly deployment in $\mathrm{M} \times \mathrm{M}$ area .We assumes a WSN with the following properties:

- It will no longer move once the sensor node deployment, and its energy is neither plentiful nor supplement.

- BS with infinite energy is deployed at the fixed position out of monitoring area.

- Every two nodes can communicate with each other, and their link is symmetrical.

- Each node has the unique ID in the network.

The same radio model as in literature [5] is used here for measuring the energy consumption of each node while communicating and data aggregating.

- The energy consumption of transmitting 1-bits to distance $d$ is given by, 


$$
E_{T x}(k, d)=\left\{\begin{array}{l}
k E_{\text {elec }}+k \varepsilon d_{f}^{2}, d<d_{0} \\
k E_{\text {elec }}+k \varepsilon_{m p} d^{4}, d \geq d_{0}
\end{array}\right.
$$

- The energy consumption of receiving l-bits is given by,

$$
E_{T x}(k, d)=k E_{\text {elec }}
$$

- While $\mathrm{d}_{0}$ is given by

$$
d_{0}=\sqrt{\varepsilon_{f s} / \varepsilon_{m p}}
$$

Where $E_{\text {elec }}$ is the transmitter/receiver electronics 'energy-expense, $d_{0}$ is the threshold distance. If the distance is less than $\mathrm{d}_{0}$, the free space model is used; otherwise, the multipath model is used. $\varepsilon_{\mathrm{fs}}$ and $\varepsilon_{\mathrm{mp}}$ represent the power amplifier energy-expense of two models. While data aggregation performed at $\mathrm{CHs}$ also needs a significant amount of energy (EDA).

\subsubsection{Zoning Division}

The division of zoning is very important in EEZCH because it represent of the clustering. Firstly we use the optimal number of $\mathrm{CH}, \mathrm{N}=\mathrm{n} * \mathrm{p}$, to divide the area into $\mathrm{m}^{2}$ zonings, where $m=$ round(N/2). And then the location information is used to find out the maximum and minimum distance between nodes and BS, $d_{\max }, d_{\min }$. Taking BS as the center of the circle, distance $\left(\mathrm{d}_{\max }-\mathrm{d}_{\min }\right) / \mathrm{m}, 2 *\left(\mathrm{~d}_{\max }-\mathrm{d}_{\min }\right) / \mathrm{m} \ldots, \mathrm{d}_{\max }-\mathrm{d}_{\min }$ as the radius to draw circles. We also introduce an auxiliary points, which is symmetrical to the base station about the $\mathrm{y}=-\mathrm{x}$ liner, to do the same work. So the area is divided into $\mathrm{m} 2$ clusters. In the end we must find out these nodes belonging to the cluster.

As shown in Fig1.1, 100 sensor nodes deployment randomly in the area of 100x100, the area is divided into 9 clusters according to above idea. It can be seen the clusters distribute uniformly in the network. This way of dividing cluster can make a range of distance from BS nodes in a cluster. 


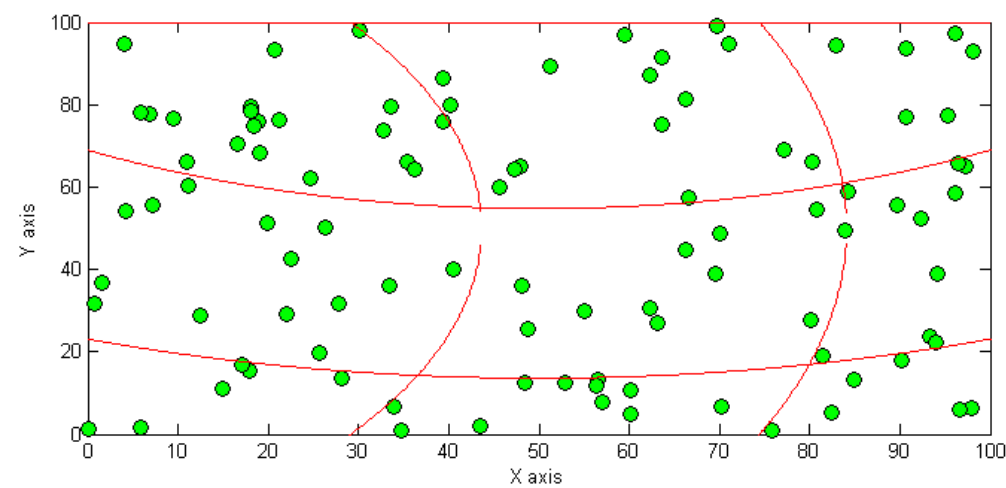

Fig. 1.1 Division of the cluster

\subsubsection{Cluster Head Selection Mechanism}

In the clustering phase, all nodes have known the one which they belong to and their distances to BS. So we can easily find out the nodes with maximum and minimum distance in a cluster, for example node $s_{i}$ and $s_{j}$. Then draw a circle with diameter $d_{i, j}$ the distance between node $\mathrm{s}_{\mathrm{i}}$ and $\mathrm{s}_{\mathrm{j}}$. If there have three nodes compete for $\mathrm{CH}$ in a cluster, as Fig. 1.2 shown, $\mathrm{s}_{\mathrm{k}}$ is inner of circle, $\mathrm{s}_{\mathrm{x}}$ is on the circle, and $\mathrm{s}_{\mathrm{y}}$ is out of circle. But which one to be the $\mathrm{CH}$ is decided by the following:

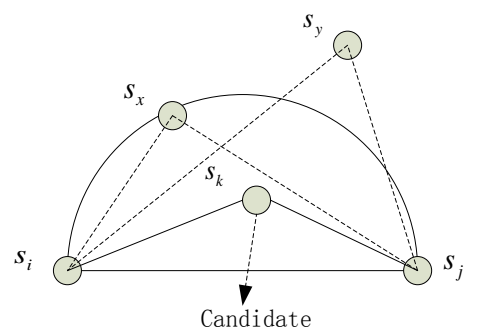

Fig. 1.2 The position of candidate in the cluster

By the triangle formula we can be obtained:

$$
D s_{i}^{2}+D s_{j k}^{2}<D_{i x}^{2} D^{2} s_{j x}^{<} \quad D_{s_{i}}
$$

While we must select the node $\mathrm{s}_{\mathrm{k}}$ with minimum distance as the candidate $\mathrm{CH}$ since the energy consumption is directly proportional to the biquadrate or square of the 
distance. And the node's residual energy is also considered. There is a threshold $t$ that is associated with the current round $r_{\text {cur }}$ and maximum simulation round $r_{\text {max }}$ :

$$
t=\left[\left(r_{\max }-r_{\text {cur }}\right) / r_{\text {max }}\right] * E_{0}
$$

$\mathrm{s}_{\mathrm{k}}$ is the real $\mathrm{CH}$ if his residual energy is more than $\mathrm{t}$ for the current round and other nodes should give up. However no any in the circle, we select the one on the circle, whose residual energy must greater than t. Finally we randomly select the one whose energy meets requirement.

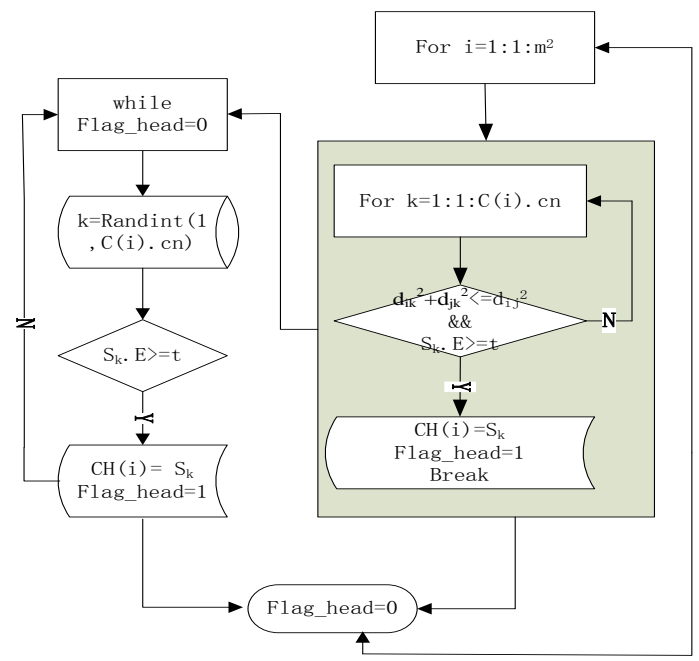

Fig. 1.3 Show the flow chart of selection $\mathrm{CH}$

\subsubsection{Data Transmission}

The data transmission phase includes the communication between the CMs and $\mathrm{CHs}$ and the communication between $\mathrm{CHs}$ and BS. The path of nodes transmission is shown in Fig. 1.4. CMs collect environmental data and sent it to their $\mathrm{CH}$ in their own time slot, and in other time slots CMs go to sleep to reduce energy consumption. In order to avoid interference and conflicts between clusters, each cluster head generates a set of spread codes, and selects one from it as its own unique identification code, and informs the nodes in the cluster. The CHs take the way of TDMA to collect the data of the nodes in the cluster, and aggregate the data for reducing the redundancy to send to the BS by single hop. 


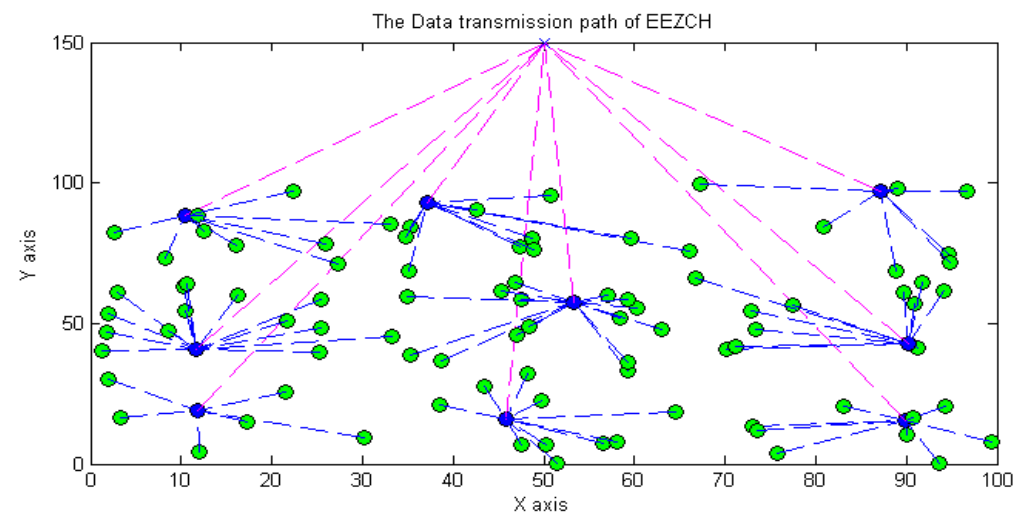

Fig. 1.4 The path of data transmission

\subsection{Simulation And Analysis}

In this section, we use MATLAB 7.1 to evaluate the performance of EEZCH compared with LEACH and LEACH-C in terms of energy-efficiency and prolonging network lifetime. We use the round to characterize the time of network operation. Specific parameter settings as shown in Table1.1:

Table 1.1 Parameters setting in simulation

\begin{tabular}{|l|l|}
\hline Parameters & Value \\
\hline Network Area & $100 \times 100(\mathrm{~m})$ \\
\hline Position of BS & $(50,150)$ \\
\hline Number of node & 100 \\
\hline $\mathrm{E}_{0}$ & $0.5 \mathrm{~J}$ \\
\hline EDA & $5 \mathrm{~nJ} / \mathrm{bit}$ \\
\hline$\varepsilon_{\mathrm{fs}}$ & $10 \mathrm{pJ} / \mathrm{bit} / \mathrm{m}^{\wedge} 2$ \\
\hline$\varepsilon_{\mathrm{mp}}$ & $0.0013 \mathrm{pJ} / \mathrm{bit}^{\mathrm{m}} \mathrm{m}^{\wedge} 4$ \\
\hline Size of packet message & $4000 \mathrm{bits}$ \\
\hline Size of control message & $100 \mathrm{bits}$ \\
\hline $\mathrm{d}_{0}$ & $87 \mathrm{~m}$ \\
\hline
\end{tabular}


Fig. 1.5 shows the total residual energy in every round over simulation time of 2500 rounds for EEZCH, LEACH, LEACH-C algorithms. The result shows that the residual energy of EEZCH is more than other two. The reason is that EEZCH makes the clustering at the begin of network, which is not to change at all rounds, while the rest of two algorithms is clustering periodically.So EEZCH can save more energy and enhance the energy efficiency.

Fig. 1.6 shows the total live nodes in every round for the three algorithms. It can be seen that nodes die out quicker using LEACH and LEACH-C algorithm than EEZCH. So the live nodes of present algorithm are more in every round.

Fig. 1.7 shows the comparison among EEZCH, LEACH, LEACH-C algorithms based on first node dead, half node dead and all node dead in the network. The data is also summarized in Table1.2. With respect to first node dead in the network EEZCH is $6.2 \%$ better than LEACH, $4.1 \%$ better than LEACH-C. Based on half node dead $\mathrm{EEZCH}$ is $26.5 \%$ better than LEACH, 23\% better than LEACH-C. EEZCH is $48 \%$ better than LEACH, $22.5 \%$ better than LEACH-C with respect to all node dead in network. Obviously, EEZCH algorithm can make the survival state of the network improved and prolong the lifetime of sensor nodes and whole network. So it easily comes to a conclusion that EEZCH is the best algorithm in the three algorithms.

Table 1.2 The comparison of dead nodes among the three algorithms

\begin{tabular}{|l|l|l|l|}
\hline & First node dead & Half node dead & All node dead \\
\hline EEZCH & 918 & 1559 & 2364 \\
\hline LEACH & 764 & 898 & 1138 \\
\hline LEACH-C & 816 & 985 & 1825 \\
\hline
\end{tabular}

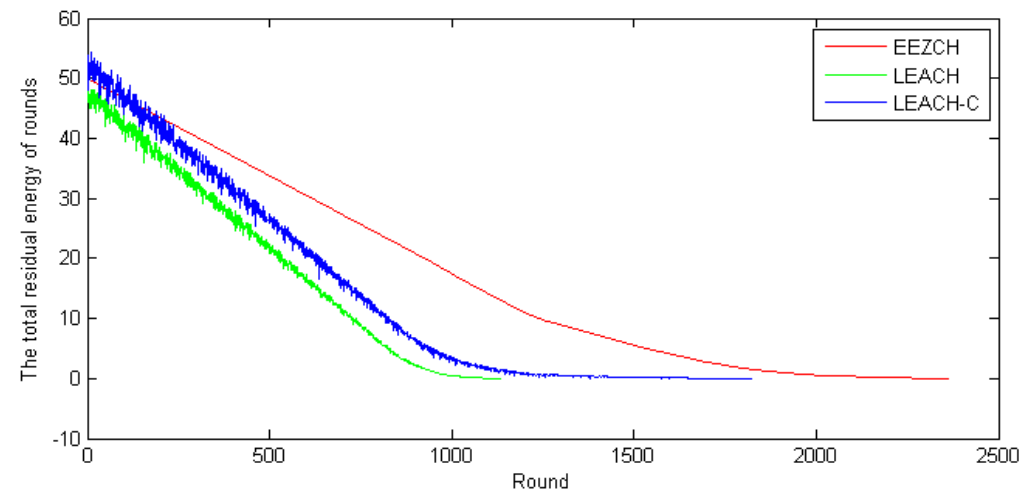

Fig. 1.5 The comparison of total residual energy 


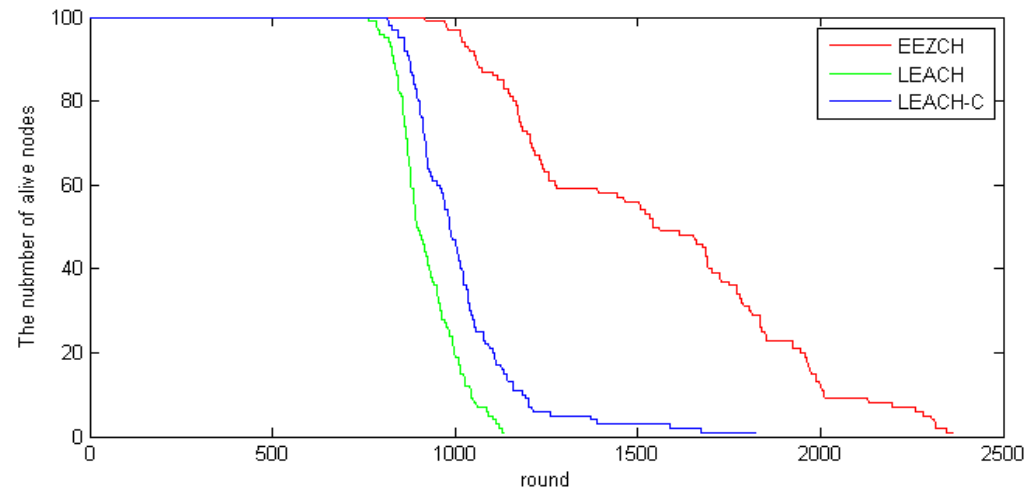

Fig. 1.6 The comparison of live nodes

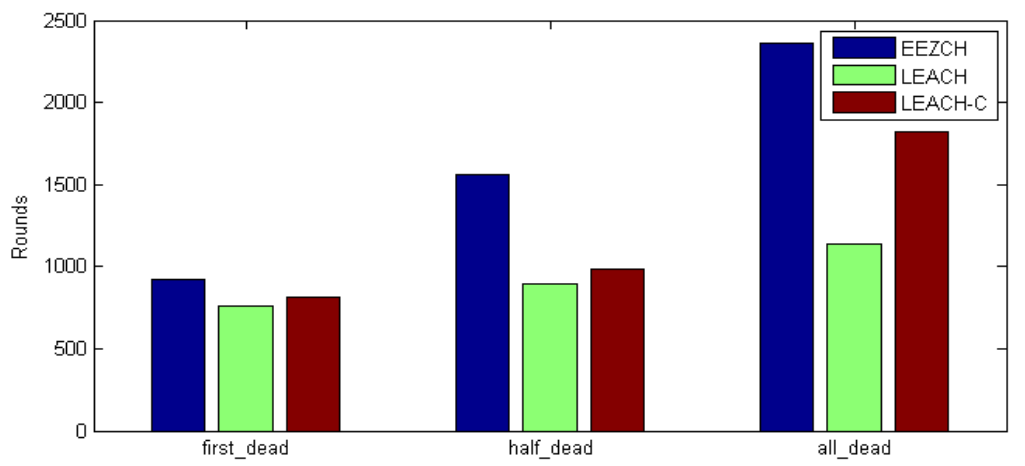

Fig. 1.7 The dead time of nodes

\subsection{Conclusions}

In this paper, we propose a novel algorithm EEZCH for WSNs. In EEZCH, we divide the monitoring area into several static clusters using the location information of node. The selection of $\mathrm{CH}$ considers nodes' residual energy and their positions in the clusters. Finally compared with the LEACH and LEACH-C algorithms, the simulation results show that the present algorithm can balance the energy consumption of all sensor nodes, enhance energy efficiency, and also extend the network lifetime. 


\subsection{Acknowledgment}

This work was supported by National Science \& Technology Major Program (2012ZX03004009), the special fund of Chongqing key laboratory (CSTC), the project of CSTC (CSTC2012jjA40044, cstc2013yykfA40010) and the project of Chongqing education committee (Kjzh11206).

\subsection{References}

1 I.F. Akyildiz, W. Su, Y. Sankarasubramaniam, and E. Cayirci, "Wireless Sensor Networks: A Survey", Computer Networks, 2002, pp. 393-442.

2 J.N.AlKarak,A.E.Kamal, "Routing techniques in wireless sensor network:a survey",IEEE Wireless Communications 11(2004)6-28.

3 A.Michail, A.Ephremides,"Energy effcient routing for connection oriented traffic in ad-hoc wireless networks",in:Proc.of the 11th IEEE International Symposium on Personal,Indoor and Mobile Radio Communications,September 2000.

4 M. Younis, M. Youssef and K. Arisha, "Energy-Aware Routing in Cluster-Based Sensor Networks", Modeling, Analysis and Simulation of Computer and Telecommunications Systems, 2002, pp. 129-136.

5 Heinzelman W R, Chandrakasan A, Balakrishnan H. Energy efficient communication protocol for wireless microsensor networks. Proceedings of the 33rd Annual Hawaii International Conference on System Sciences (HICSS'00): Vol 1, Jan 4-7, 2000, Maui, HI, USA. Los Alamitos, CA, USA: IEEE Computer Society, 2000: 14p

6 W.B.Heinzelman,A.P.Chandrakasan and H.Balakrishnan,"An application Specific Protocol Architecture for Wireless Microsensor Netwo-rks", IEEE Transaction on Wireless Communications, 2002, pp.660-670.

7 S. Lindsey, C.Raghavendra, "PEGASIS: Power-Efficient GAthering in Sensor Information Systems," In Proc. IEEE Aerospace Conference,USA, Montana, 2002, Vol. 3, pp. 1125-1130.

8 O. Younis and S. Fahmy, "Heed: a hybrid, energy-efficient, distributed clustering approach for ad hoc sensor networks," Mobile Computing,IEEE Transactions on, vol. 3, no. 4, Oct.-Dec. 2004, pp. 366-379.

9 Younis O, Fahmy S. Heed: A hybrid, energy-efficient, distributed clustering approach for ad-hoc sensornetworks[J]. IEEE Trans. On mobile Computing, 2004, 3(4):660-669.

10 Manjeshwar A, Grawal DP. TEEN: A protocol for enhanced efficiency in wireless sensor networks[C].In: Proc. of the 15th Parallel and Distributed Processing Symp. San Francisco, 2001, pp: $2009-2015$. 